\title{
Ureaplasma Urease Genes have Undergone a Unique Evolutionary Process
}

\author{
Hiromi Nishida ${ }^{*}$
}

\begin{abstract}
Agricultural Bioinformatics Research Unit, Graduate School of Agricultural and Life Sciences, University of Tokyo, 11-1 Yayoi, Bunkyo-ku, Tokyo 113-8657, Japan
\end{abstract}

\begin{abstract}
Ureaplasma, a member of mycoplasmas, has a unique ATP synthesis system, which is coupled to the urea hydrolysis. Urease catalyzes the hydrolysis of urea into carbon dioxide and ammonia. Phylogenetic analyses of the urease genes indicated that Ureaplasma urease genes were not gained by recent horizontal transfer and have a unique evolutionary process. Ureaplasma unique ATP synthesis system leaded to breakdown of the glycolysis pathway. Some glycolytic genes are absent and some glycolytic genes are evolving under relaxed selection in Ureaplasma. Probably glycolytic genes can be used as an indicator of ATP synthesis system. Thus, the organisms that have incomplete glycolysis pathway or glycolytic genes evolving under relaxed selection would have an ATP synthesis system independently of the glycolysis.
\end{abstract}

Mycoplasmas are widespread in nature as parasites of mammals, reptiles, fishes, arthropods, and plants [1]. During the mycoplasma evolution, gene loss has occurred frequently, resulting in very small genome size [1-3]. The reductive evolution of mycoplasmas is still in progress. The genus Ureaplasma is a member of mycoplasmas, which generates $95 \%$ of its ATP using the hydrolysis of urea [4]. Growth of Ureaplasma is dependent on urea [5]. This unique ATP synthesis is not found in the other mycoplasmas. In fact, key enzymes in the glycolytic pathway are absent in Ureaplasma [6]. In addition, some glycolytic genes of Ureaplasma are evolving under relaxed selection [7, 8]. Thus, the glycolysis pathway is collapsing in Ureaplasma. is coupled to ATP synthesis in Ureaplasma [9]. This unique system of Ureaplasma leaded to breakdown of the glycolysis pathway. For example, Ureaplasma does not have any genes encoding glucose-6-phosphate isomerae [10]. It suggests that the glycolysis system had been important for ATP synthesis rather than glucose metabolism at least in Ureaplasma (Fig. 1). Generally the TCA (tricarboxylic acid) cycle is linked to the glycolysis pathway, which has played an important role in ATP synthesis of many organisms and conserved in the course of evolution. On the other hand, the urea hydrolysis is not linked to the glycolysis pathway. Ureaplasma generates ATP through the urea hydrolysis not through the glycolysis. Probably Ureaplasma glycolysis pathway was not able to be

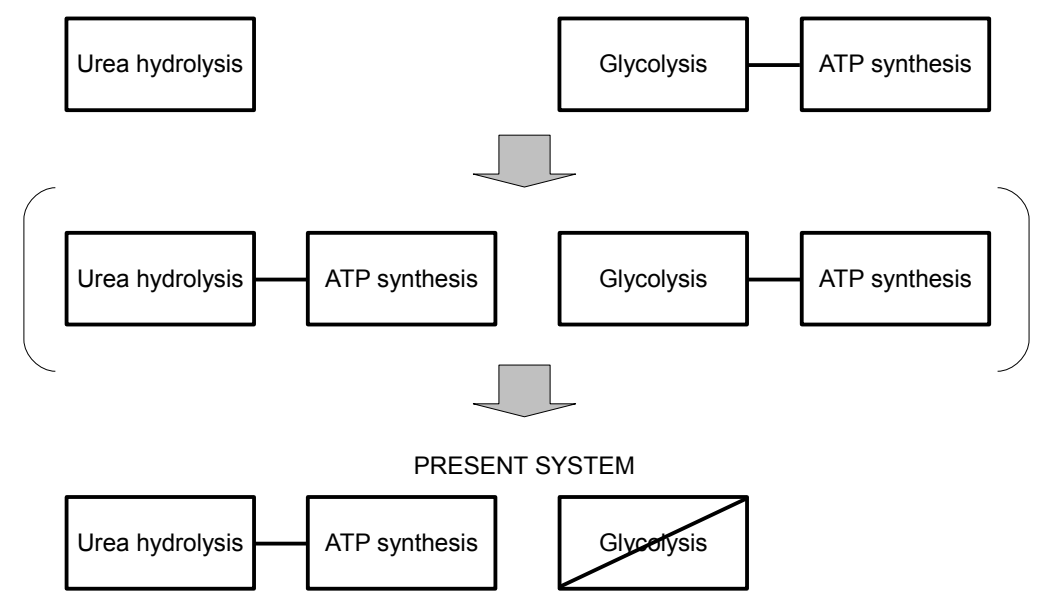

Fig. (1). Model of change of ATP synthesis system in Ureaplasma. In Ureaplasma, the ATP synthesis through the glycolysis had been changed to that through the urea hydrolysis. The glycolysis pathway was broken after the urea hydrolysis was coupled to ATP synthesis.

Urea is hydrolyzed into carbon dioxide and ammonia in many organisms. However, it is unique that the urea hydrolysis

*Address correspondence to this author at the Agricultural Bioinformatics Research Unit, Graduate School of Agricultural and Life Sciences, The University of Tokyo, 1-1-1 Yayoi, Bunkyo-ku, Tokyo 113-8657, Japan;

E-mail: hnishida@iu.a.u-tokyo.ac.jp broken before the urea hydrolysis was coupled to ATP synthesis. Therefore, after the coupling of the urea hydrolysis and ATP synthesis, dominant ATP synthesis had been changed from through the glycolysis to through the urea hydrolysis during the Ureaplasma evolution (Fig. 1). 
(A)

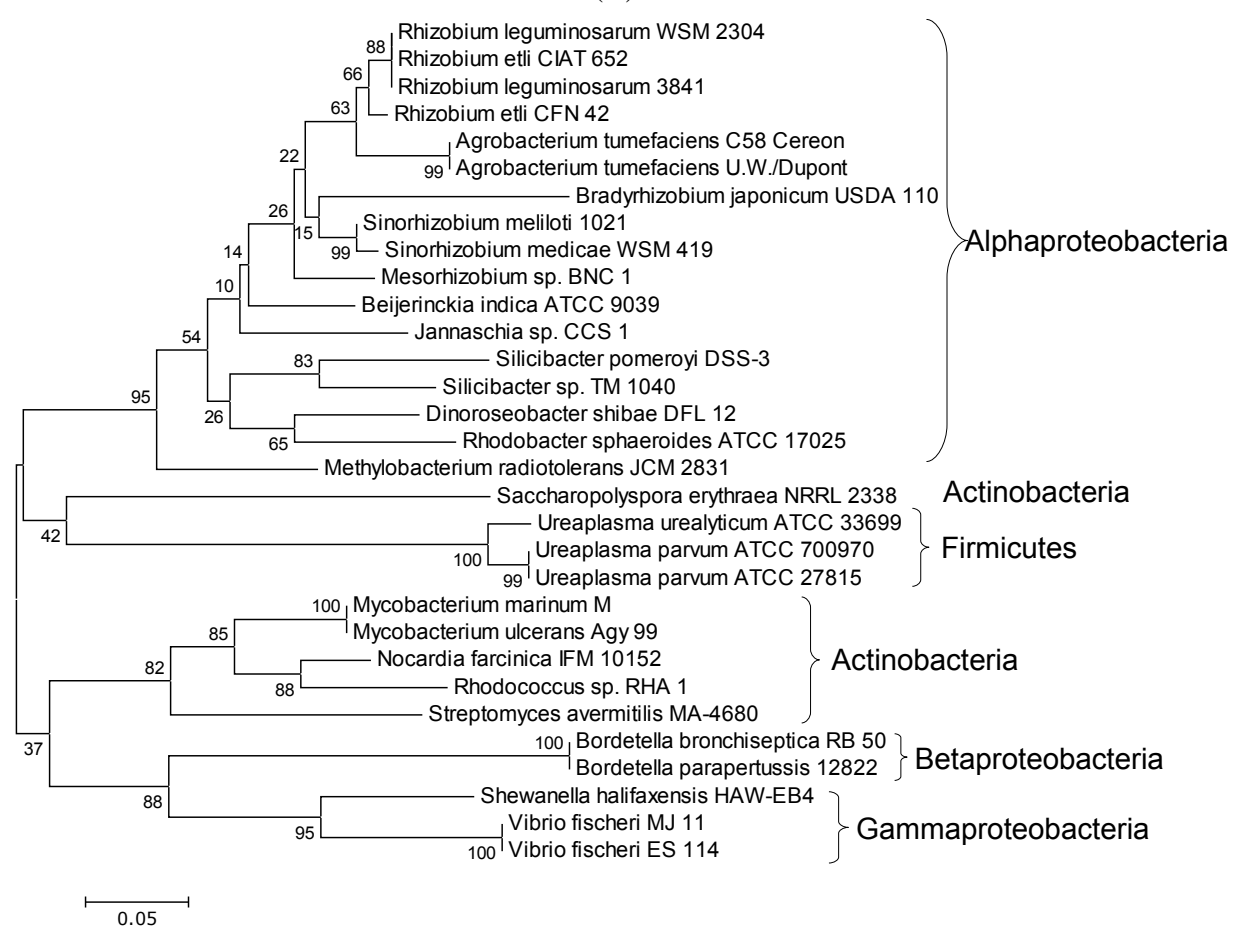

(B)

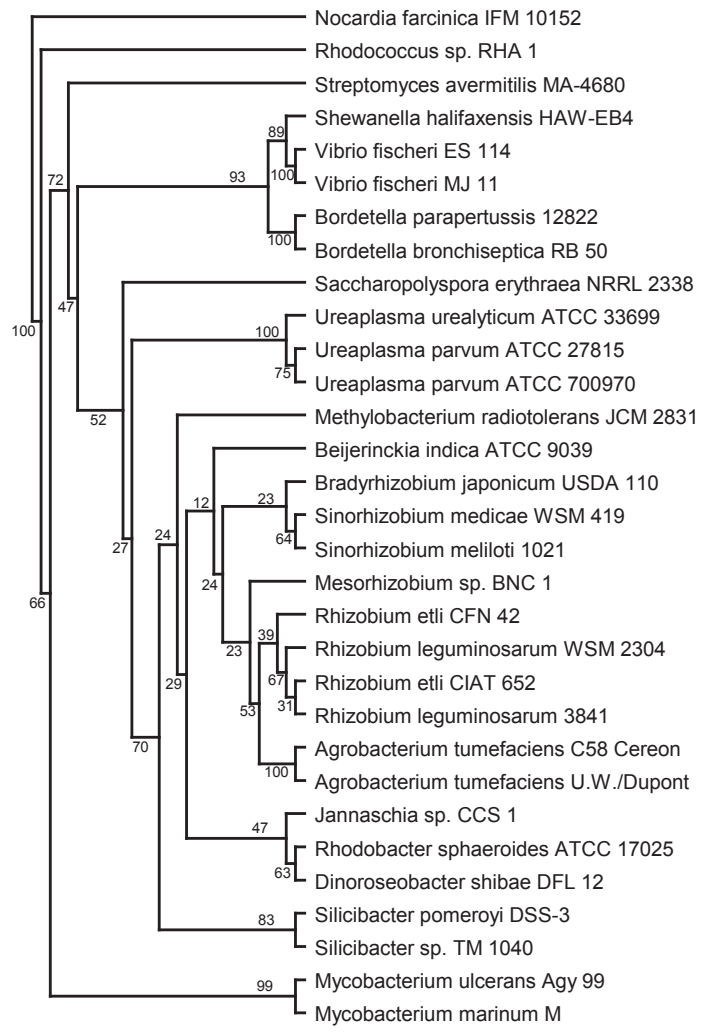

Fig. (2). Phylogenetic relationships among Ureaplasma UreA and the homologues. (A) Neighbo r-joining tree. The BLAST program was used to search the GenomeNet website for proteins homologous to Ureaplasma UreA with E-value < $10^{-23}$. Ureaplasma UreA and 30 homologous proteins were multiple aligned using the CLUSTAL W program. Phylogenetic tree was reconstructed, based on the multiple-alignment with complete deletion of gap sites using the neighbor-joining method of MEGA software with 1000 bootstrap replicates. (B) Maximum likelihood tree. Phylogenetic tree was reconstructed using the maximum likelihood method of the PHYLIP program with 100 bootstrap replicates. The JTT model was used as the model of amino acid substitution. Number of times to jumble in the PROML program was 2. 
(A)

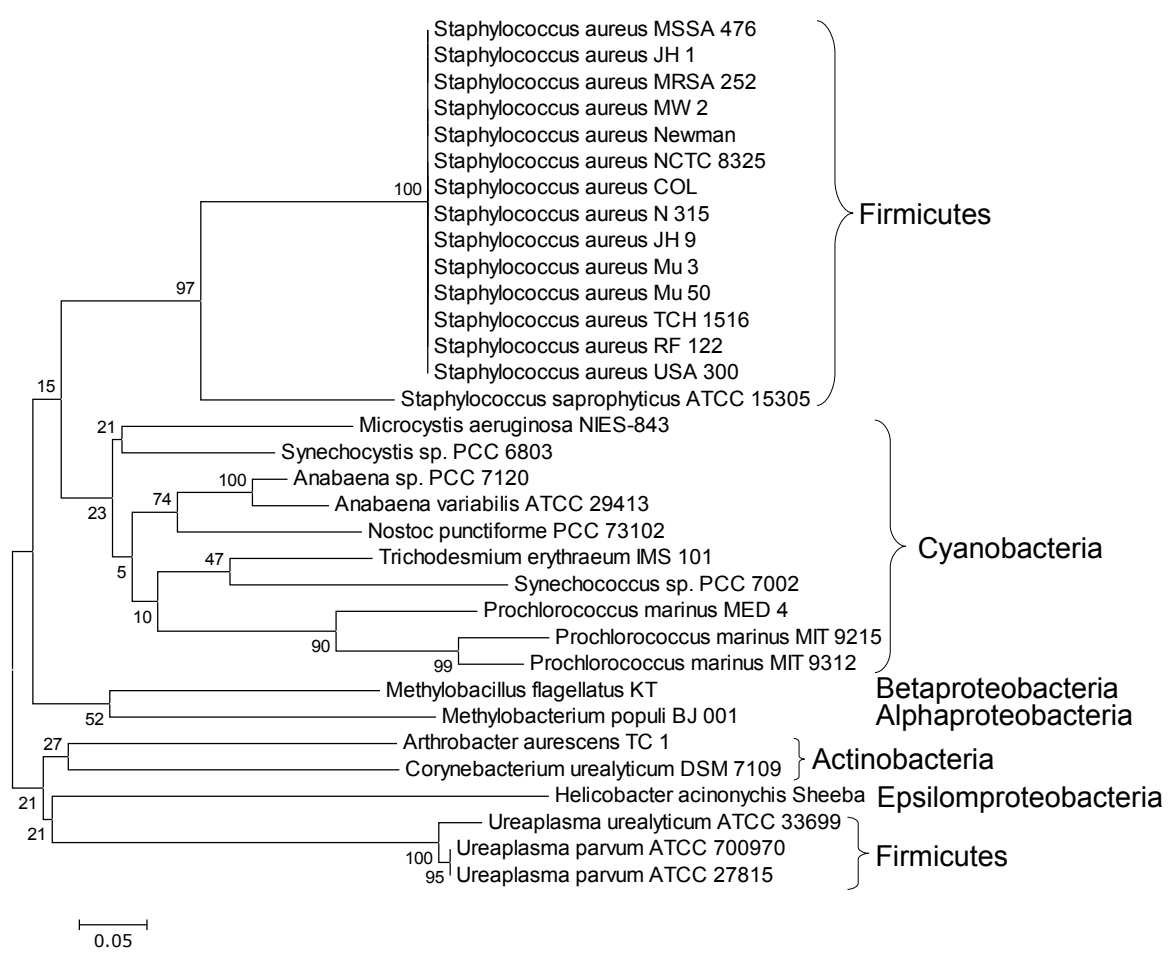

(B)

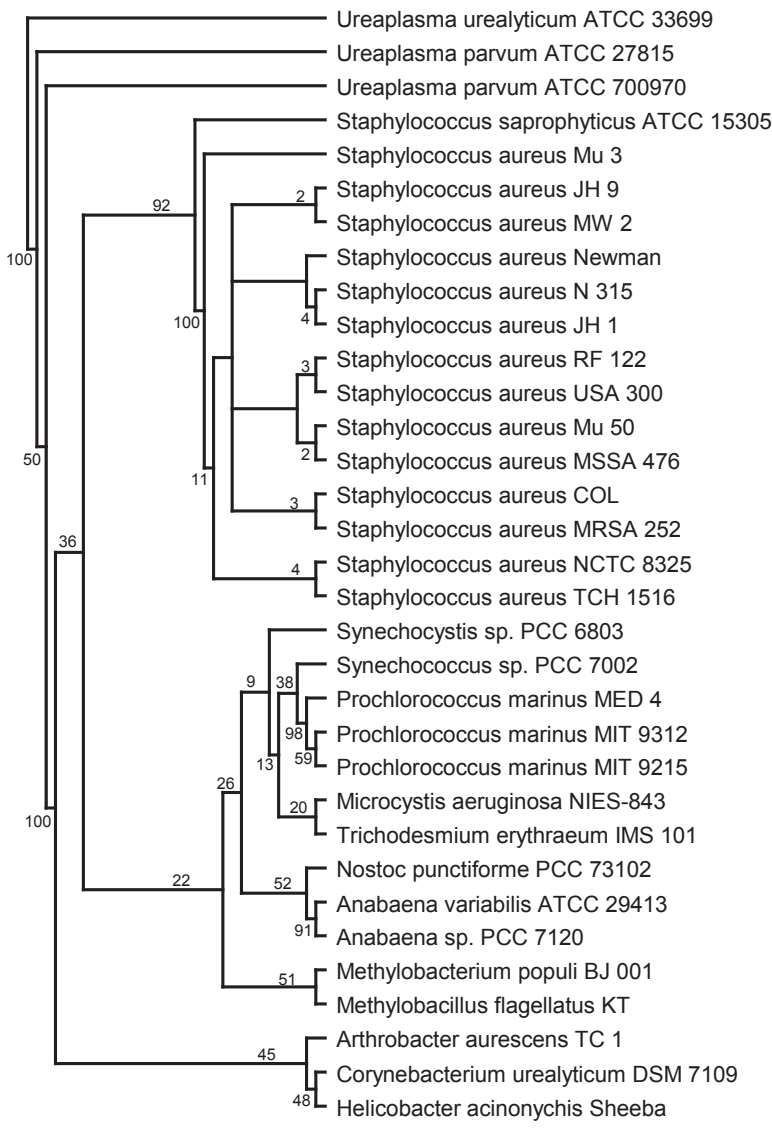

Fig. (3). Phylogenetic relationships among Ureaplasma UreB and the homologues. (A) Neighbor-joining tree. The BLAST program was used to search the GenomeNet website for proteins homologous to Ureaplasma UreB with E-value $<10^{-25}$. Ureaplasma UreA and 32 homologous proteins were multiple aligned using the CLUSTAL W program. Phylogenetic tree was reconstructed, based on the multiplealignment with complete deletion of gap sites using the neighbor-joining method of MEGA software with 1000 bootstrap replicates. (B) Maximum likelihood tree. Phylogenetic tree was reconstructed using the maximum likelihood method of the PHYLIP program with 100 bootstrap replicates. The JTT model was used as the model of amino acid substitution. Number of times to jumble in the PROML program was 2 . 
(A)

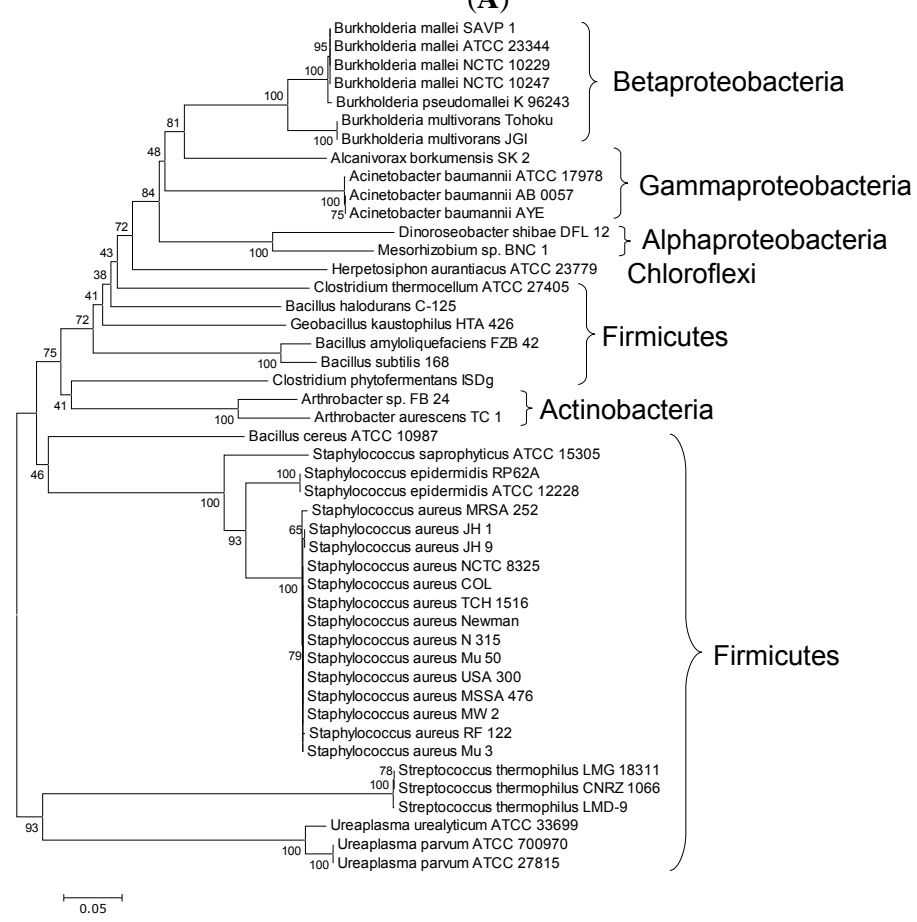

(B)

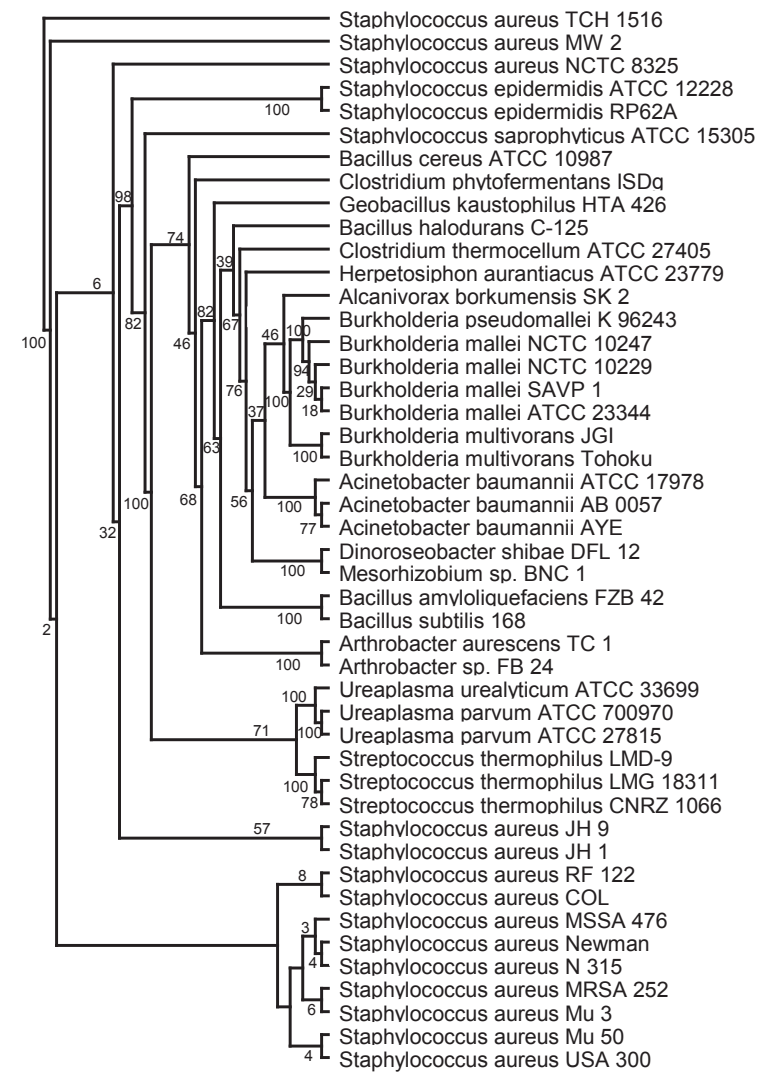

Fig. (4). Phylogenetic relationships among Ureaplasma UreC and the homologues. (A) Neighbor-joining tree. The BLAST program was used to search the GenomeNet website for proteins homologous to Ureaplasma UreC with E-value $<10^{-170}$. Ureaplasma UreA and 45 homologous proteins were multiple aligned using the CLUSTAL W program. Phylogenetic tree was reconstructed, based on the multiplealignment with complete deletion of gap sites using the neighbor-joining method of MEGA software with 1000 bootstrap replicates. (B) Maximum likelihood tree. Phylogenetic tree was reconstructed using the maximum likelihood method of the PHYLIP program with 100 bootstrap replicates. The JTT model was used as the model of amino acid substitution. Number of times to jumble in the PROML program was 2 . 
UreD

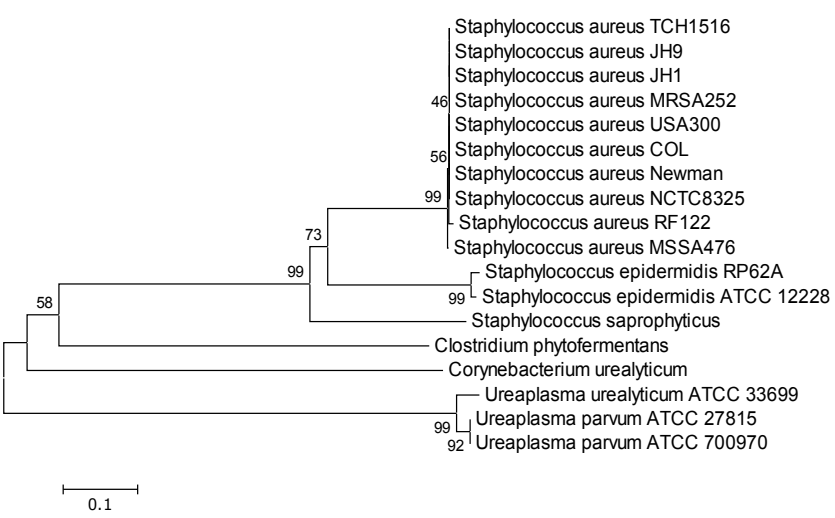

UreF

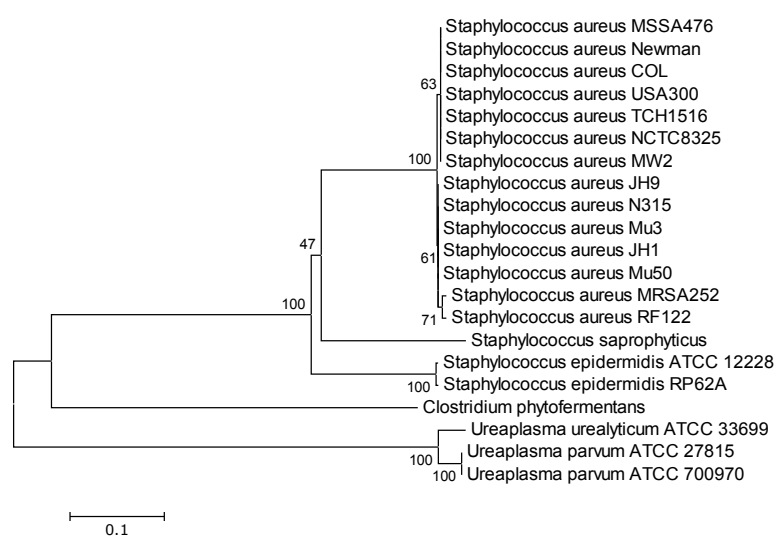

UreE

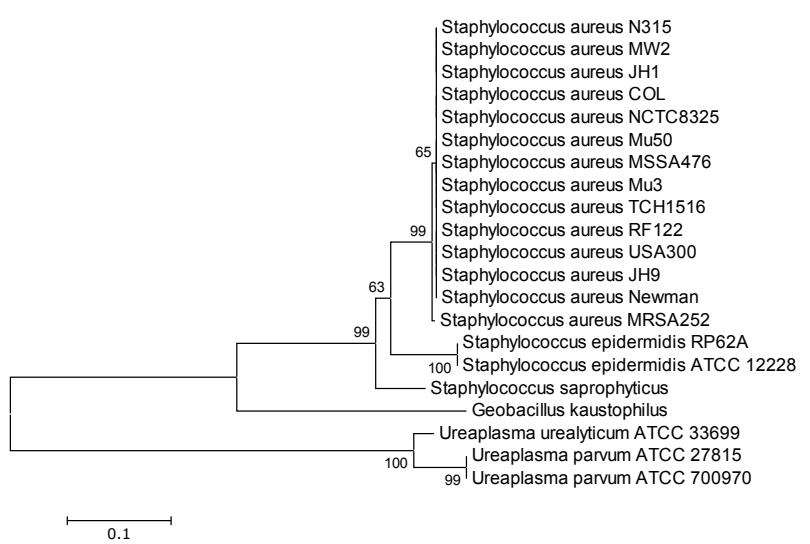

UreG

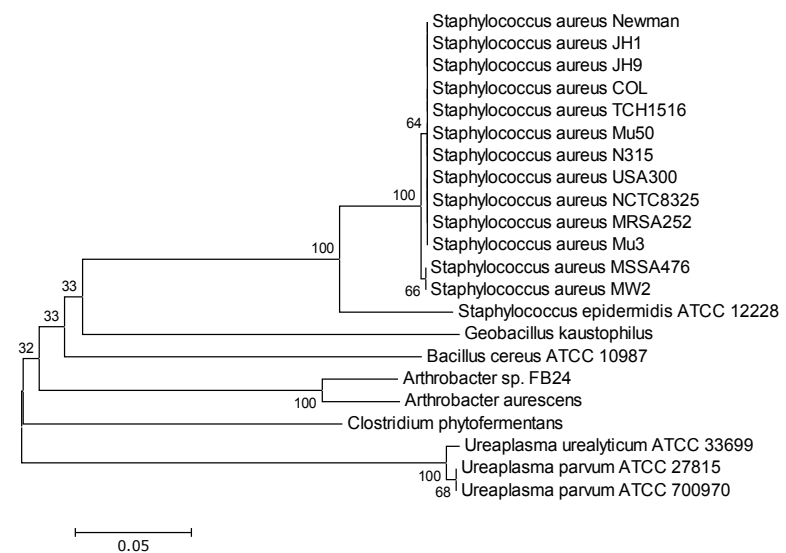

Fig. (5). Phylogenetic relationships among Ureaplasma UreD and the homologues, UreE and the homologues, UreF and the homologues, and UreG and the homologues. The BLAST program was used to search the GenomeNet website for proteins homologous to Ureaplasma UreD, UreE, UreF, and UreG of Ureaplasma parvum ATCC 27815 with $E$-value $<10^{-35}, 10^{-25}, 10^{-40}$, and $10^{-77}$, respectively. This search led to identification of 17 UreD homologues, 20 UreE homologues, 20 UreF homologues, and 21 UreG homologues. The 18 UreD, 21 UreE, 21 UreF, and 22 UreG proteins were multiple aligned using the CLUSTAL W program on the GenomeNet website. Phylogenetic tree was reconstructed, based on the multiple-alignment with complete deletion of gap sites using the neighbor-joining method of MEGA software with 1000 bootstrap replicates.

Urease catalyzes the hydrolysis of urea into carbon dioxide and ammonia. Urease has three core subunits, UreA, UreB, and UreC. Generally these three core subunits and the related accessory proteins UreD, UreE, UreF, and UreG are clustered on the genome. The order of the Ureaplasma (belongs to the Firmicutes) urease genes in the cluster is identical to that of the Bacillus sp. TB-90 (belongs to the Firmicutes) urease genes [11], suggesting that an ancestor of the Firmicutes had the urease gene cluster. Some species had lost some urease genes during the evolution. For example, Bacillus subtilis contains urease structural genes but lacks the accessory genes typically required for the maturation of urease [12]. On the other hand, the mycoplasmas except for Ureaplasma completely lack urease genes. I have some questions. Was the Ureaplasma urease genes (gene cluster) transferred horizontally? Or did the mycoplasmas except for Ureaplasma lose urease genes in the course of the mycoplasma evolution? Did the Ureaplasma urease have a unique evolutionary process? In order to answer these questions, I compared the amino acid sequences of urease genes of Ureaplasma with the homologous proteins in this study.

The BLAST program was used to search the GenomeNet website (http://www.genome.jp) for proteins homologous to UreA, UreB, UreC, UreD, UreE, UreF, and UreG of Ureaplasma parvum ATCC 27815 with $E$-value $<10^{-23}, 10^{-25}, 10^{-170}$, $10^{-35}, 10^{-25}, 10^{-40}$, and $10^{-77}$, respectively. This search led to identification of 30 UreA homologues, 32 UreB homologues, 45 UreC homologues, 17 UreD homologues, 20 UreE homologues, 20 UreF homologues, and 21 UreG homologues. The 31 UreA, 33 UreB, 46 UreC, 18 UreD, 21 UreE, $21 \mathrm{UreF}$, and $22 \mathrm{UreG}$ proteins were multiple aligned using the CLUSTAL W program on the GenomeNet website. Phylogenetic trees were reconstructed, based on the multiple-alignments with complete deletion of gap sites using the neighbor-joining method of MEGA software [13] with 1000 bootstrap replicates. In addition, phylogenetic trees of UreA, UreB, and UreC proteins were reconstructed, based on the multiple alignments with complete deletion of 


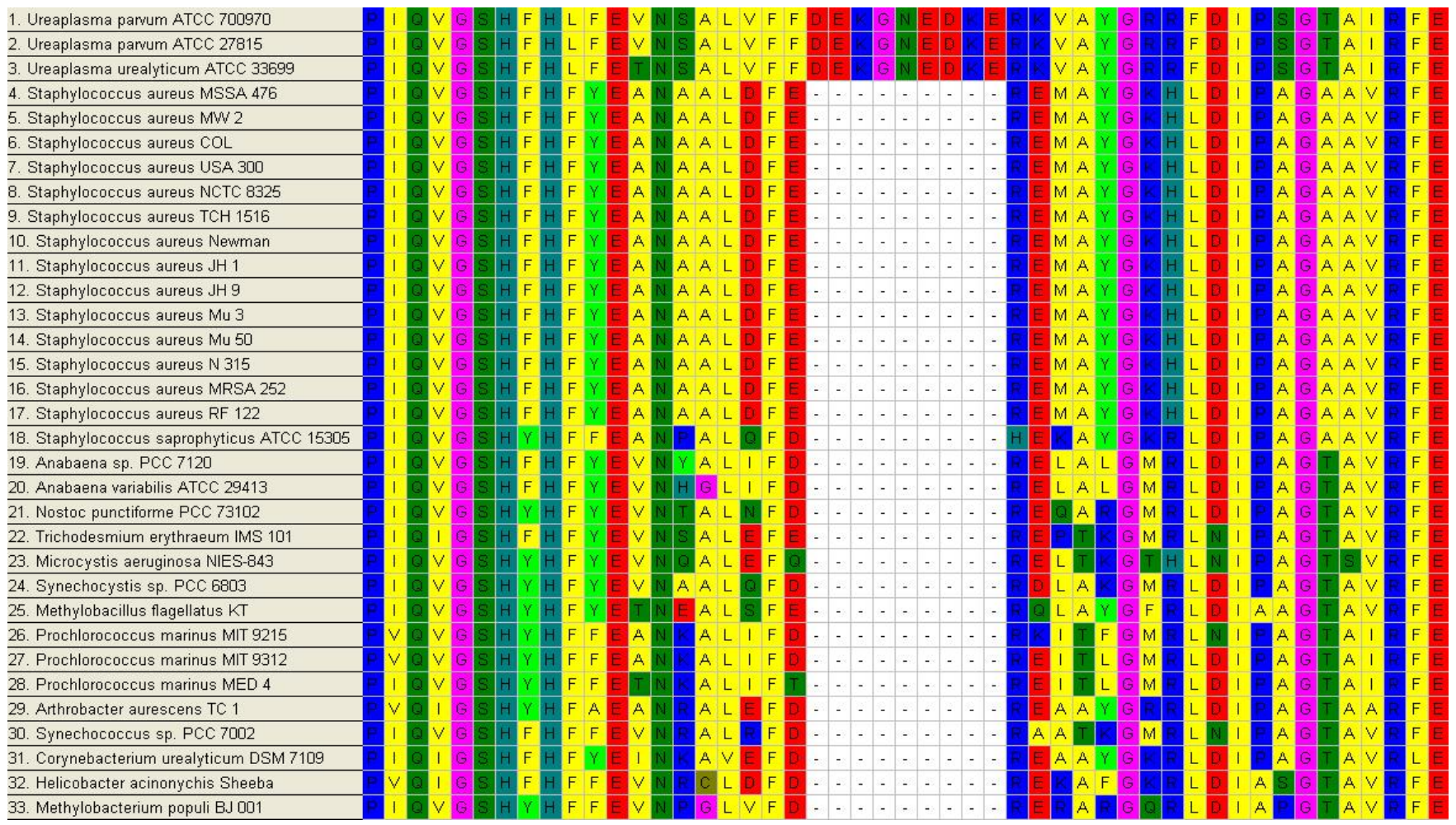

Fig. (6). Ureaplasma UreB specific amino acids insertion region. Ureaplasma has a specific 9-amino acids insertion (positions 65-73) in the multiple alignment of $33 \mathrm{UreB}$ proteins.

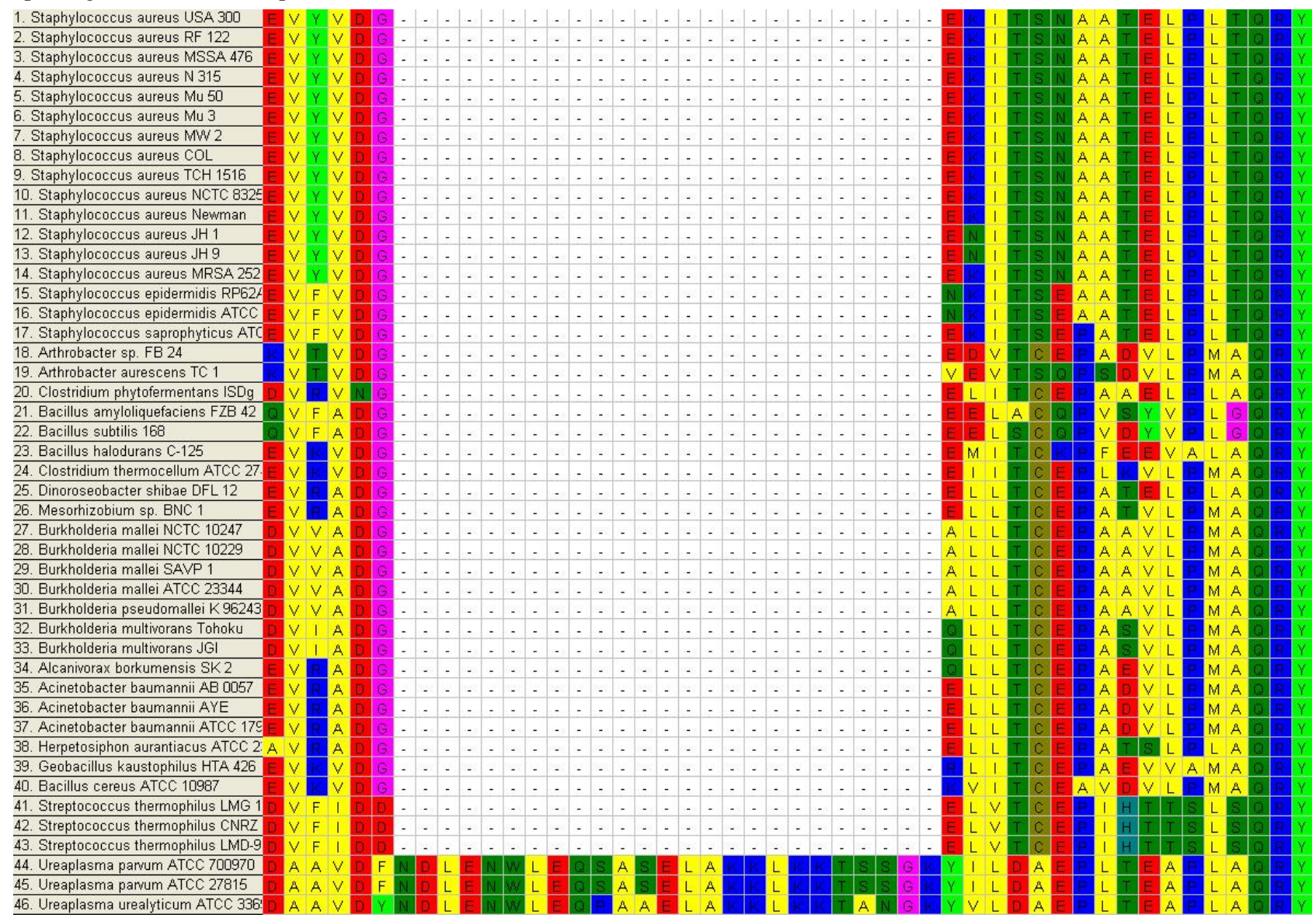

Fig. (7). Ureaplasma UreC specific amino acids insertion region. Ureaplasma has a specific 25-amino acids insertion (positions 554-578) in the multiple alignment of $33 \mathrm{UreC}$ proteins. 
gap sites using the maximum likelihood method of the PHYLIP program (http://evolution.genetics.washington.edu/ phylip.html) with 100 bootstrap replicates. The JTT model was used as the model of amino acid substitution. Number of times to jumble in the PROML program was 2.

The phylogenetic analyses showed that the KEGG database [14] used in this study did not have closely related protein to UreA and UreB of Ureaplasma (Figs. 2A, B and 3A, B). On the other hand, Ureaplasma UreC is closely related to Streptococcus UreC with $93 \%$ bootstrap support in the neighbor-joining tree (Fig. 4A) and $71 \%$ support in the maximum likelihood tree (Fig. 4B). The diverging points of UreA, UreB, and UreC of Ureaplasma are very deep in the neighbor-joining trees (Figs. 2A, 3A, 4A), strongly suggesting that Ureaplasma urease genes (gene cluster) were not gained by recent horizontal transfer. It was also supported by the phylogenetic trees of urease accessory proteins (Fig. 5). Interestingly, Ureaplasma UreB and UreC have Ureaplasma-specific amino acids insertions (Figs. 6, 7). Thus, Ureaplasma urease gene cluster has a unique evolutionary process and Ureaplasma has a unique urea hydrolysis system coupling to ATP synthesis. Maybe Ureaplasma ancestor had used urease in order to live in the urea-rich environment. During the evolution, the urea hydrolysis was coupled to ATP synthesis system. After the event, glycolysis pathway has not been essential for ATP synthesis in Ureaplasma. Some glycolytic genes are absent and some are evolving under relaxed selection in Ureaplasma. Probaly the establishment of the unique ATP synthesis system triggered those changes. If so, glycolytic genes can be used as an indicator of ATP synthesis system. Thus, the organisms that have incomplete glycolysis pathway or glycolytic genes evolving under relaxed selection would have a unique ATP synthesis system independently of the glycolysis.

\section{REFERENCES}

[1] Razin S, Yogev D, Naot Y. Molecular biology and pathogenicity of mycoplasmas. Microbiol Mol Biol Rev 1998; 62: 1094-156.

[2] Jaffe JD, Stange-Thomann N, Smith C, et al. The complete genome and proteome of Mycoplasma mobile. Genome Res 2004; 14: 1447-61.

[3] Oshima K, Kakizawa S, Nishigawa H, et al. Reductive evolution suggested from the complete genome sequence of a plantpathogenic phytoplasma. Nat Genet 2004; 36: 27-9.

[4] Smith DGE, Russell WC, Ingledew WJ, Thirkell D. Hydrolysis of urea by Ureaplasma urealyticum generates a transmembrane potential with resultant ATP synthesis. J Bacteriol 1993; 175: 3253-8.

[5] Kenny GE, Cartwright FD. Effect of urea concentration on growth of Ureaplasma urealyticum (T-strain mycoplasma). J Bacteriol 1977; 132: 144-50.

[6] Pollack JD. Ureaplasma urealyticum: an opportunity for combinatorial genomics. Trend Microbiol 2001; 9: 169-75.

[7] Oshima K, Nishida H. Phylogenetic relationships among mycoplasmas based on the whole genomic information. J Mol Evol 2007; 65: 249-58.

[8] Oshima K, Nishida H. Detection of the genes evolving under Ureaplasma-specific selection. J Mol Evol 2008; 66: 529-32.

[9] Romano N, La Licata R, Russo Alesi D. Energy production in Ureaplasma urealyticum. Pediatr Infect Dis 1986; 5: S308-12.

[10] Glass JI, Lefkowitz EJ, Glass JS. The complete sequence of the mucosal pathogen. Ureaplasma urealyticum. Nature 2000; 407: 757-62.

[11] Neyrolles O, Ferris S, Behbahani N, Montagnier L, Blanchard A. Organization of Ureaplasma urealyticum urease gene cluster and expression in a suppressor strain of Escherichia coli. J Bacteriol 1996; 178: 647-55.

[12] Kim JK, Mulrooney SB, Hausinger RP. Biosynthesis of active Bacillus subtilis urease in the absence of known urease accessory proteins. J Bacteriol 2005; 187: 7150-54.

[13] Tamura K, Dudley J, Nei M, Kumar S. MEGA4: Molecular Evolutionary Genetics Analysis (MEGA) software version 4.0. Mol Biol Evol 2007; 24: 1596-99.

[14] Kanehisa M, Araki M, Goto S, et al. KEGG for linking genomes to life and the environment. Nucleic Acids Res 2008; 36: D480-D484. 